\title{
DEL CONFLICTO AL POSTACUERDO: UN PROCESO EDUCATIVO
}

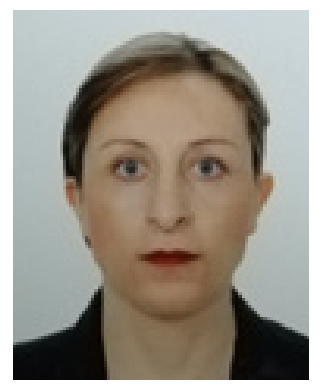

Fecha de recepción: 06/10/2018
Olga Piedad Aguirre Salazar ${ }^{1}$

Institución Educativa Bello Horizonte de la ciudad de Medellín, Magister en Educación Católica de Manizales, Colombia olgapiedad.as@gmail.com

Una sociedad sin formación política y que no entiende que los modelos de convivencia son creados por las personas, puede llegar a creer que la violencia es un destino.

José Bernardo Toro, 2002:40.

\section{RESUMEN}

Este ensayo académico, aborda la construcción de ciudadanía como eje para consolidar una cultura de paz, teniendo como premisa que para escindir las raíces de la violencia es fundamental y necesario la transformación de las nuevas generaciones, con propuestas pedagógicas que se validen en los contextos y ámbitos, como ejercicios democráticos, éticos y responsables. El artículo inicialmente aduce las tensiones políticas que se encuentran en la actual fase de la implementación de los acuerdos de paz, entre las fuerzas que tiene en vilo una posible reconciliación. Realza la importancia de los procesos escolares de niños y jóvenes como parte de la estrategia transformadora, en donde uno de los componentes que hay que incorporar en el currículo escolar es el fortalecimiento de la formación sociopolítica. Primero, conociendo la historia reciente, y segundo, admitiendo que los valores públicos cambiarían las lógicas obtusas de la excluyente democracia colombiana que le dieron origen a la confrontación armada. La importancia de las acciones educativas que se emprendan para que la cultura para la paz quede instalada en la escuela, se encuentra en función de crear las condiciones para superar los vacíos que desde la legislación educativa pueda encontrar y en consecuencia se pueda trasmitir a la sociedad.

Palabras clave: Conflicto, Postacuerdo, Implementación, Proceso educativo.

\footnotetext{
${ }^{1}$ Docente de química de la Institución Educativa Bello Horizonte de la ciudad de Medellín, Magister en Educación Católica de Manizales, Candidata a Doctora de la Universidad Metropolitana de Educación, Ciencia y Tecnología de la República de Panamá.

olgapiedad.as@gmail.com
} 


\title{
FROM THE CONFLICT TO THE POST-ACOORD: AN EDUCATIONAL PROCESS
}

\begin{abstract}
This academic essay addresses the construction of citizenship as the axis to consolidate a culture of peace, based on the premise that to transform the roots of violence, the transformation of new generations is essential, with pedagogical proposals that are validated in contexts and areas, as democratic, ethical and responsible exercises. The article initially adduces the political tensions that are in the current phase of the implementation of the peace accords, among the forces that have in suspense a possible reconciliation. It highlights the importance of school processes for children and young people as part of the transformative strategy, where one of the components that must be incorporated into the school curriculum is the strengthening of socio-political education. First, knowing the recent history, and second, admitting that public values would change the obtuse logics of the exclusive Colombian democracy that gave rise to the armed confrontation. The importance of the educative actions that are undertaken so that the culture for peace is installed in the school, is in function of creating the conditions to overcome the gaps that from the educational legislation can find and consequently can be transmitted to the society .
\end{abstract}

Keywords: Conflict, Postaccords, Implementation, Educational process.

\section{INTRODUCCIÓN}

La realidad colombiana vive actualmente una oportunidad política y social sin precedentes, el fin de la violencia política con uno de los brazos armados de los grupos de izquierda. En consecuecia, la sociedad colombiana en el escenario del postacuerdo, tiene que trascender de los actos simbólicos avenidos con el reciente proceso de paz, hacia una transformación ciudadana que modifique la actual axiología política; ese capital humano, permeado por la nueva realidad, deberá ser el insumo de una propuesta pedagógica, que lógicamente protagonizará las nuevas generaciones.

La escuela, como instrumento de desarrollo social y de cambio deberá validar sus procesos formativos, a fin de promover un pensamiento autónomo, con matiz crítico y reflexivo, que no solo madure en la situación, sino que esté preocupado por la recuperación de la memoria histórica y de la apropiación de discursos de mayor intensidad argumentativa para apostarle a las nuevas demandas sociales.

Los acuerdos suscritos, como resultado de la confluencia y conciliación de puntos de vista que antes del actual momento eran antagónicos y en pugna, se convierten ahora, en un recurso educativo, potencialmente significativo para lograr el propósito establecido, formar futuros ciudadanos activos, conscientes de su papel y de su capacidad participativa como actores sociales, protagonistas de su devenir histórico, y que conocen, piensan, reflexionan, y apoyan las salidas negociadas a un panorama que se había vuelto casi inamovible en nuestra 
sociedad, la guerra y la violencia como formas no solo cotidianas, sino naturales de la vida nacional.

\section{EL ESCENARIO}

Al concluir las negociaciones entre el Estado y las Farc-Ep con la suscripción de los acuerdos, la terminación del conflicto en Colombia parece una quimera con un desenlace sombrío. Las posturas acérrimas de quienes se han opuesto a este proceso lograron que la votación en el plebiscito de 2016 no fuera positiva, y, posteriormente en las elecciones presidenciales de junio de 2018 ascendiera al poder la derecha radical que rebatía los compromisos logrados, entre ellos la Justicia Especial para la Paz (JEP) como realidad legal. La JEP, le otorga soporte jurídico a toda las acciones propias del conflicto, al investigar, esclarecer y juzgar a quienes hicieron parte de él, como una forma de enfrentar la verdad, reparar a las víctimas y atacar la impunidad por la senda de la reconciliación.

Trascender del acto simbólico de la firma de los Acuerdos a la implementación de estos, es un compromiso que no sólo los organismos del Estado tendrán que cumplir, en general, la sociedad será veedora y garante para que llegue a buen término. Es claro que para cerrar este periodo de violencia deba existir un compromiso nacional y más cuando se tiene un capital humano joven que empieza a cuestionar el panorama político tradicional y demanda otras lógicas ante esta geopolítica de la desesperación. Una paz tan esquiva requiere voluntad política y compromiso social, además de acciones coherentes en torno a la justicia, la equidad y participación democrática. Es en este momento en el que la función transformadora de la escuela debe verse reflejada en sus procesos educativos.

\section{LOS JÓVENES}

Colombia, un país que tiene alrededor de 13 millones de jóvenes según el Departamento Nacional de Estadística, Dane, aporta un potencial electoral significativo a los 36.227.267 de personas habilitadas para votar según el último informe de la Registraduría (2018). Con estas características, las nuevas generaciones se constituyen en sustrato para dar respuesta a las urgencias sociales en torno a las acciones y las responsabilidades de lo público y constituirse en actores protagonistas de esta parte de la historia. Imposible olvidar 'La Marcha Estudiantil del Silencio' por el magnicidio de Luis Carlos Galán Sarmiento en 1989, una iniciativa popular liderada por los jóvenes condenando el crimen. Génesis para la transformación de las bases políticas unos años después con la denominada 'Séptima Papeleta', que despejaría el camino para la Constitución Política de Colombia de 1991, demostrando de este modo la necesidad de cambio que exigían los jóvenes ante la crisis de las instituciones del Estado y su incapacidad transformadora.

A 25 años de escribirse la constitución del 91, se firman los Acuerdos entre el Estado y las Farc, sobre este hecho Nussbaum expresa: "[...] en un momento así, es muy posible que haya bastante incertidumbre, puesto que muchas posturas diferentes compiten por la 
aceptación de la gente" (Nussbaum, agosto 25 de 2016), tal como quedó demostrado con el impensado triunfo del $\mathrm{NO}$ en el plebiscito. Este resultado mueve a los jóvenes a respaldar la paz, y por tercera ocasión se recoge la herencia de Gaitán con 'La Marcha del Silencio' a favor de la no violencia. Inicia de este modo un camino con muchas cortapisas hacia la transformación de una sociedad más plural, equitativa y justa.

La escuela, como se lo tiene que proponer, debe estar en medio de tal frustración nacional avivando con más propuestas concretas y con menos retórica mendaz los cambios demandados en sus aulas. No es para menos, la formación de nuevas ciudadanías más conscientes de su devenir histórico, y de su importante rol social, exige un trabajo de sumo compromiso político en las aulas, porque es desde este escenario que se refundarán las bases para construir esa sociedad anhelada a través de proyectos cohesionadores que le den el impulso a la educación que lleve a mejorar los niveles de argumentación y análisis en medio de tanta verbosidad vacua y manipuladora.

\section{LA ESCUELA}

Considerar otras subjetivaciones políticas en la juventud, a fin de lograr compromisos ciudadanos éticamente responsables en la comunidad, implica que desde la escuela se promueva un pensamiento crítico reflexivo, se recupere la memoria histórica, y se conquiste el poder comunicativo, Hoyos expresa que: "La democracia es la síntesis del principio discursivo con la forma del derecho" (2002: 10). Elementos que configuran el discurso político en las aulas y el empoderamiento activo que catapulta al estudiante a las acciones de participación democráticas. De tal modo que se le de paso a una verdadera cultura ciudadana al «construir una paz estable y duradera» tal como lo establecía la pregunta del plebiscito. Esto lleva a valorar las condiciones actuales de la estructura del currículo escolar en Colombia y su direccionamiento hacia una cultura de paz y la construcción de ciudadanía. Y, en otra instancia, reconocer las propuestas juveniles que buscan hacer pedagogía a fin de dinamizar desde otros escenarios la implementación de los Acuerdos.

Es oportuno recordar que a partir de 1994 las clases de historia desaparecieron del currículo escolar, dejando en el olvido los pasajes de la vida nacional que tanto signaron lo que hoy somos como nación. Actualmente, las pedagogías de la memoria están siendo incorporadas en las prácticas pedagógicas más desde las voluntades individuales de los maestros que como política educativa. La escuela debe sensibilizar a los niños y jóvenes frente a una realidad que dejó millones de personas vulneradas a lo largo de tantos años de conflicto, una forma de resistir y enfrentar esas causas que lo hacen perdurar. No obstante, la formación sociopolítica no es exclusiva de una disciplina en particular y por ende todo el proceso educativo debe nutrirse desde las distintas perspectivas de conocimiento.

Los Estándares básicos de Competencias ciudadanas. Formar para la ciudadanía... ¡Sí es posible!, son directrices emanadas por el Ministerio de Educación Nacional en 2004, que buscan enfatizar la formación ciudadana: “...como un proceso que se puede diseñar, con base 
en principios claros, implementar, con persistencia y rigor, evaluar continuamente e involucrar en los planes de mejoramiento de cada institución" (Estándares básicos, 2004: 5). Las competencias ciudadanas están agrupadas bajo tres tópicos: la convivencia y paz, la participación y responsabilidad democrática y la pluralidad, y la identidad y valoración de las diferencias. Al no ser considerada asignatura dentro del currículo, estas competencias se convierten en compromiso no sólo de la institución sino de la comunidad educativa en general al entregar los elementos para reconocer al otro como interlocutor auténtico y al lenguaje como mediador universal.

La organización conceptual de los estándares no menciona el tema de los procesos de paz, y los acuerdos son equiparados al concepto de normas que un grupo o comunidad establece, tal como aparece entre los grados cuarto y quinto, en el eje sobre Convivencia y Paz: "Reconozco el valor de las normas y los acuerdos para la convivencia en la familia, en el medio escolar y en otras situaciones” (Estándares básicos, 2004: 18). Ante tal vacío conceptual y en el marco de la implementación de los acuerdos, el Ministerio nuevamente se pronuncia y reglamenta la Cátedra de la Paz, para garantizar una educación pensada en las actuales circunstancias: "crear y consolidar un espacio para el aprendizaje, la reflexión y el diálogo de la cultura de la paz y el desarrollo sostenible” (Ley 1732, Parágrafo $2^{\circ}$ ).

La Cátedra de la Paz está organizada por Proyectos bajo las siguientes líneas: diversidad natural y cultural, resolución de conflictos, prevención del acoso escolar y memoria histórica. Las variables asociadas al proceso no fueron contempladas en la presente cátedra y los acuerdos, propiamente la historia de estos, aparecen como un tema disgregado de libre desarrollo curricular. Lo que evidencia el grado de improvisación que tienen los contenidos propuestos con la realidad vivida durante el proceso de la negociación.

Desconcierta que La cátedra de la Paz no haya sido masivamente socializada con el magisterio para presentar el alcance de la misma, y cuestiona que en el equipo de autores tampoco haya sido convocado algún maestro en ejercicio que le imprimiera elementos de la realidad escolar, rural o urbana. Extrañamente el Acuerdo final tampoco es incluido como material de estudio en ese tránsito hacia la reconciliación, desconociendo que los Acuerdos tienen como objetivo eliminar las raíces de la violencia política y que por tanto guardan unos aprendizajes significativos inherentes a todo el proceso: diálogos, negociaciones y soluciones, y a la concreción de los acuerdos en los derechos humanos, los valores democráticos, las identidades culturales. Quizá el logro más importante sea aprender que las salidas negociadas son las medidas para solucionar los conflictos, y los modelos de justicia el instrumento de acatamiento para los responsables de la guerra.

Era trascendental llamar a la escuela porque en ella también confluyó lo más absurdo de la naturaleza humana, la violencia. Y, ¿Cómo no llamarla cuando presenció el horror que vivieron tantos seres?, ¿ ¿Acaso hay que continuar asistiendo al engaño al reconocerla como un espacio en el que se debe acceder al conocimiento como condición sine qua non de niños y jóvenes abstractos y universales? Es imperativo hablar de las escuelas reales en plural, 
una que desafía e incluso es blanco de la crudeza del conflicto y resiste, y la otra que ampara a aquellos que optaron por huir de un destino fatal a otro no menos doloroso. A ese último grupo van las familias o lo que queda de ellas con sus hijos para salvaguardar su integridad abandonando sus tierras, y también van los maestros, que truncan un proyecto político social al sobreponer su seguridad ante el miedo que enajena, y al tener vulnerado su derecho al trabajo.

Quien tiene que abandonar todo lo que ha construido por presiones de la guerra, afronta una de las más duras batallas, reconstruir su cultura e identidad en lugar ajeno a sus raíces. A la escuela le corresponde desde el quehacer pedagógico favorecer la incorporación de estos niños y jóvenes con demandas tan particulares producto de experiencias traumáticas y eliminar los estigmas que los revictimice. Además, superar la desidia social traducida en una falta de solidaridad que insiste en la violencia como referente cultural, para que no sea más fácil justificar la barbaridad de los grupos en confrontación que sentir empatía con estas personas que sufren, y a los que nadie parece importar, pero que engrosan la ya larga lista de desplazamiento interno en Colombia, que según Acnur es de las más altas del planeta.

Las personas que se quedaron en sus regiones, vivieron en carne propia la violación permanente de sus derechos humanos. Nadie responderá por el acecho permanente de la tragedia, pero en la memoria quedarán intactas las incursiones a la propiedad privada que terminaron en masacres y asesinatos selectivos en presencia de esposas, madres e hijos. Padres que al oponerse al reclutamiento forzado de sus hijos, fueron asesinados en su presencia. Mujeres y niñas violentadas sexualmente. Es por esto que la memoria histórica en este o en cualquier otro contexto de terror es testigo irrebatible de quienes perdieron la voz, para no repetir la ignominia o para que en algún momento pueda existir perdón y reconciliación.

Muchas escuelas continuaron ahí, marcadas con tinta indeleble tras su invasión, convertidas en cuarteles improvisados, iqué paradoja! en un escenario de vida. Y sus alrededores sembrados, no con el esfuerzo del campesino, sino con un asesino silencioso que se unió a la guerra, las minas antipersona. El infortunio acabará con miles de humanidades, dejará mutilados, ocasionará todo el pánico para no volver a poner los pies allí. Después de esto empezarán a reconstruir sus vidas tras un largo proceso que sane las heridas físicas y morales.

Rural o urbana, la escuela continuará, tendrá que llegar con más fuerza, con acciones concretas en la reconstrucción del tejido social, no será fácil recuperar la confianza pero no existe otra vía. El cambio de gobierno por uno de derecha y su reiterada oposición al proceso de paz, permite entrever que el camino que se inicia para la transformación hacia una renovada sociedad más plural, equitativa y justa, tendrá muchas trabas. La escuela tendrá que validar acciones que mejoren su calidad y el acceso a la cultura, para desmontar desde allí con argumentos contundentes las mentiras que obnubilan cualquier entendimiento frente a la realidad nacional. 
Respecto a proyectos de liderazgo juvenil inspirados en el postacuerdo, es de resaltar el denominado Fortalecimiento a Iniciativas Juveniles de la Fundación Paz y Reconciliación, Pares, cuya estrategia central se basa en potenciar el emprendimiento y la capacidad de organización, mediante talleres vocacionales, culturales y deportivos. (Pares, 2018). PAZ a la página de la Red Kolumbien, tiene como propósito abordar a la comunidad sobre los temas centrales del acuerdo, mediante el análisis crítico y reflexivo, fue premiada por el Alto Comisionado para la Paz con la mención: Promoción del diálogo intergeneracional en la construcción de Paz en el marco del programa Este es tú reto (Domopaz, 2017).

Este es tú reto, una propuesta del Alto Comisionado para Paz, correspondiente a la Red Territorios de Paz que busca experiencias de jóvenes entre 14 y 28 años cuyo propósito central es sensibilizar sobre el proceso de paz y dinamizar los acuerdos suscritos, haciendo apropiación de los recursos tecnológicos y de la capacidad creativa. En el desarrollo de este proyecto se presentan experiencias de todo el territorio nacional, en el 2018 está en la segunda versión. (Alto Comisionado para la Paz, 2016). Las estrategias y propósitos son diversos, se destacan proyectos que buscan recuperar memoria histórica, sensibilizar a los padres y madres jóvenes, narrativas culturales, actividades de reconciliación, integración de territorios, el cuerpo como territorio de paz, y otras de tipo deportivo y cultural.

La Fundación Centro Internacional de Educación y Desarrollo humano, Cinde, apoya con sus programas los procesos de construcción de paz en Colombia, mediante actividades formativas: Niños, niñas y jóvenes Constructores de Paz en Cocorná Antioquia; Vacaciones recreativas en Constructores de Paz; Educación, ciudadanía y paz en María la Baja y el Carmen de Bolívar; Red de Consejos de niños, niñas y adolescentes; Sentidos y prácticas políticas de niños, niñas y jóvenes en contextos de vulnerabilidad; Narrativas de paz: niños, niñas y jóvenes sobrevivientes de la guerra; Constructores de paz en zona rural de Manizales (cinde.org.co). La fundación tiene recopiladas otras experiencias en el libro, Experiencias Alternativas de acción política con participación de jóvenes en Colombia (Cinde, 2011). Los jóvenes serán protagonistas de las conquistas sociales a partir de la refundación de valores democráticos, asentada desde la capacidad discursiva como interés común societal y en la aceptación del otro como interlocutor válido.

...precisamente por ello pensamos que vale la pena apostarle en la formación ciudadana al tipo de imperativos propios de la filosofía práctica, en el reino del deber ser, no "en el plano de la lucha burocrática e institucional, sino en el de una hipotética sociedad que quiere guiarse por valores democráticos" (Palacios, 1999: 62).

Colombia es un país de esperanza, y de jóvenes indignados que deben entender que en sus manos está la reconstrucción de este país para que respondan públicamente como protagonistas que son de la historia reciente. Cuando esto ocurra se tendrá la prueba de la coherencia entre los propósitos de la escuela y los de Nación, y, por primera vez los adultos podrán entender que la escuela forma sociopolíticamente a sus ciudadanos como principio y esencia de su gestión y no agota hasta el fracaso contenidos que para la actual sociedad perdieron hace tiempo relevancia. Los jóvenes deben construir discursos propios con la tutela de sus maestros, deben recuperar la palabra arrebatada y llenarla de tal fervor patriótico como el que despierta el equipo de fútbol nacional. 


\section{LECCIONES APRENDIDAS DEL PROCESO DE PAZ}

La importancia que tienen los jóvenes en el actual panorama político nacional, demanda el conocimiento de dos hechos que cambiaron las lógicas guerreristas entre el Estado y la insurgencia de la Farc-Ep: El Proceso y la suscripción de los Acuerdos. Respecto al primer concepto, se toma la definición de Vicenç Fisas por su compromiso investigativo alrededor del mundo, y como director de Escola de Cultura de Pau de la Universidad de Barcelona, y conocedor del proceso desarrollado en Colombia:

Se entiende por procesos de paz todos aquellos esfuerzos, especialmente políticos y diplomáticos, destinados a resolver los conflictos armados o los conflictos que actualmente no están en fase armada, pero que en el pasado sí lo fueron y todavía necesitan negociaciones para llegar a un acuerdo satisfactorio entre las partes. (Fisas, 2016, p. 15).

La parte final del proceso de paz es la firma de los acuerdos. El acuerdo general de paz lo suscribe el equipo negociador, conformado por las partes implicadas con acompañamiento y asesoría internacional. En él se pone de realce todas las circunstancias que le dieron origen al conflicto y las que se derivaron de este. Queda plasmado en un documento oficial como resultado final de la negociación. Fisas, lo describe como: "un esfuerzo para lograr un acuerdo que ponga fin a la violencia, así como para implementarlo, mediante negociaciones que pueden requerir la mediación de terceros" (2010: 11). El acuerdo no es el fin del conflicto, es considerada como la última etapa de la negociación.

El momento del acuerdo es el más icónico, porque en él se condensa el esfuerzo que en un periodo significativo logró desmantelar las subjetividades de cada una de las partes negociadoras para convertirlas en oportunidades de transformación para un país. A la escuela le corresponde promover una verdadera cultura para la paz que aliente procesos de mediación frente a los conflictos escolares y la no repetición como acto profundo de contrición, $\mathrm{y}$, en consecuencia, atomizar las falsedades de los medios de comunicación del imaginario social instaladas en la conciencia colectiva. Este proceso de reconstrucción demanda contenidos más relevantes al desarrollo de los sucesos actuales, es por esto que los acuerdos, se constituyen en oportunidad para lograr aprendizajes propios y presentes.

El primero de los acuerdos es el de la Reforma Rural Integral, y no es para menos, la violencia nace en el campo por la inequidad en la tenencia de la tierra, y las fracasadas reformas agrarias que sumieron al campesino en la exclusión y el abandono, dejando la explotación desmedida del suelo y su usufructo en manos de personas que adquirieron poder económico y político, lo que en la actualidad sigue invariante. De ahí la trascendencia que tiene la educación, no sólo la rural, en la construcción de ciudadanías pensadas sobre la base de la dignidad, la autonomía y la defensa de los territorios, para creer que en algún momento de la historia se cerrarán las brechas, y la equidad con justicia social no será una utopía. 
La participación política es el segundo punto tras la urgencia de incluir otras voces en el debate político y llevar a otro nivel la confrontación, al pasar de la fuerza a la palabra en el ejercicio pleno de la democracia participativa. Contradictorio que el exterminio de los líderes sociales se haya exacerbado luego de la firma de los acuerdos, líderes que en su mayoría están reclamando sus tierras o exigiendo la sustitución de cultivos, tal como ocurrió en su momento con los miembros de la Unión Patriótica, lo que ensombrece la inclusión política en detrimento de ciudadanías activas y conscientes de su devenir histórico. El tercer punto, El fin del conflicto, se apoya en el acuerdo anterior, en el sentido de dejar las armas para lograr la reincorporación a la vida civil y optar por salidas negociadas a través de mecanismos transparentes, confiables y creíbles, en lugar de la reducción violenta del adversario.

La lucha contra las drogas es quizá el punto más álgido de todo el proceso de negociación, por el impacto que ha generado en la sociedad una economía criminal de la dimensión del narcotráfico y las tres esferas en las que hay que dirigir las acciones en su contra: producción, distribución y consumo. Lo que exige políticas contundentes para la restitución de cultivos con su respectivo control y seguimiento y todas aquellas dirigidas al consumo como problema de salud pública que penetra todas las estructuras de la sociedad, incluyendo a la escuela donde los tentáculos del microtráfico la tienen asolada. Lo que lleva a un inicio temprano en el consumo de drogas y otros riesgos derivados como la explotación sexual y la participación en hechos delictivos para su consecución. El costo social es alto e impacta el proyecto de vida de jóvenes que cada vez inician a más temprana edad su consumo, incluyendo abandono del sistema escolar.

El quinto punto, Sistema integral de verdad, justicia, reparación y no repetición, pone a prueba la madurez de una sociedad como la colombiana acostumbrada a dirimir sus conflictos en forma violenta. Este punto busca resarcir el dolor acumulado durante tantos años de crueldad con las víctimas y se centra en el perdón y no en el olvido, y en el restablecimiento de sus derechos. En este apartado las pedagogías de la memoria cobran un valor inusual, y deben provocar reflexiones acompañadas de acciones que modifiquen las dinámicas sociopolíticas a fin de desnaturalizar la violencia, además, desarrollar un trabajo conjunto entre la escuela y sus comunidades en aras de promover nuevas subjetivaciones políticas que alienten procesos de participación democrática.

Los acuerdos buscan el restablecimiento de los derechos de las víctimas, otorgándole a las Farc-Ep la posibilidad de participar democráticamente en la vida política y la búsqueda de una identidad nacional a través del desarrollo rural integral. La escuela tiene como encargo dirimir los conflictos a través del diálogo, un aprendizaje para el ciudadano del postacuerdo acostumbrado a la intolerancia y la exclusión. Exhorta al perdón y a la reconciliación desde una postura individual para nada condescendiente, al enfrentar la verdad, lo que desde la formación sociopolítica debe ser efectivamente reavivado. Nussbaum a propósito de este nuevo escenario político, insta por las nuevas formas de la educación: 
[...] un compromiso con el fortalecimiento de los valores humanos necesita abarcar tanto el currículo y la pedagogía, dando a los jóvenes la capacidad del pensamiento crítico y la argumentación respetuosa, impartiendo entendimiento de un amplio rango de perspectivas sociales e históricas y también de cultivar la capacidad con las obras de arte, la literatura y la música. El estudio de la filosofía, la literatura y las artes no es inútil: es de importancia urgente, puesto que todas las personas, cualquiera que sea su trabajo futuro, serán ciudadanos, responsables por el futuro del pacto social (Nussbaum, agosto 25 de 2016).

Para García (1993), el efecto de un proceso de paz consiste en posibilitar la construcción de democracias donde los Derechos Humanos se preservan y respetan. La experiencia de la negociación deja como realidad la paz o eso se espera que ocurra, pero hay unos absolutos de paz que también quedan como aprendizaje: la justicia, la libertad y la equidad, cuya aplicación se hace extensiva a otras situaciones de la vida. Una negociación malograda, implica la derrota de ambos bandos. Un posible escenario de negociación integra conversaciones, diálogos, y, la negociación como tal, otorga los elementos para que la escuela reevalúe sus procesos de mediación.

\section{CONCLUSIONES}

La academia tiene un saldo en rojo con la sociedad colombiana, enfrascada en teorías, pasa por alto su función política y beneficia indirectamente los intereses clientelistas de los más poderosos, lo que acentúa los males de la población más vulnerable que repite una y otra vez sus historias de abuso y exclusión. Cambiar esa racionalidad es una tarea que la escuela está en mora de hacer, para reconciliar las diferencias y consolidar un proyecto de Nación.

La naturalización del conflicto es tan familiar y necesaria para una significativa parte de los colombianos, que, abrir la posibilidad de transformación hacia una cultura de paz, impresiona, a tal punto, de preferir los planteamientos bélicos que han hecho de la guerra un negocio que deja generosos ingresos por sobre la vida de miles, en lugar de promover una sociedad más equitativa y justa para todos. Extraña que al desaparecer uno de los actores armados más poderosos del escenario, se pretenda asignar a defensa una partida del presupuesto nacional tan alta como la correspondiente a un escenario en guerra para el año 2019.

La implementación de los acuerdos pasa por un momento álgido, al llegar al poder la extrema derecha, férrea opositora a este proceso de reconciliación. Tal circunstancia en lugar de acrecentar el pesimismo con todo lo que puede pasar con los procesos de transformación social, debe movilizar las conciencias ciudadanas y continuar con lo que conducirá a una paz estable y duradera, una forma de resistencia ciudadana frente a la brutalidad de la confrontación armada. 


\section{REFERENCIAS BIBLIOGRÁFICAS}

- Alto Comisionado para la Paz. (2016). Este es tú reto. Disponible en:

- http://www.altocomisionadoparalapaz.gov.co/Prensa/Paginas/2016/junio/ Convocatoria-Este-es-tu-reto.aspx.

- Cinde. Categoría: Jóvenes. Disponible en:

- https://www.cinde.org.co/sitio/resultado_busqueda. php?d=j\%F3venes $+\&$ Submit2=.

- Dane. (2018). Reloj de población. Disponible en: http://www.dane.gov.co/reloj/, Junio 21 de 2018

- Domopaz (2017). PAZa la Página. Disponible en: http://www.domopaz.org/ item/47-premio-paza-la-pagina.

- Fisas, V. (2010). ¡Ato el fuego! Manual de procesos de paz. Barcelona: Ed. Icaria.

- Fisas, V. (2016). Anuario de procesos de paz. Barcelona: Ed. Icaria.

- Fundación Paz y Reconciliación. (2018). Iniciativas juveniles. Disponible en: http://pares.com.co/2018/04/12/iniciativas-juveniles/.

- García, C. (1993). Lecciones históricas aprendidas de los procesos de negociación para la paz en algunos países del mundo. Disponible en: https://revistas.uniandes. edu.co/doi/pdf/10.7440/histcrit7.1993.02.

- Hoyos, G. (2002). Camino hacia nuevas ciudadanías. Bogotá: Departamento Administrativo de Bienestar Social del Distrito.

- Ley N 1732. Congreso de la República de Colombia, Bogotá, 01 de septiembre de 2014. Disponible en: http://wsp.presidencia.gov.co/Normativa/Leyes/Documents

- Ministerio de Educación Nacional (2004). Estándares básicos de Competencias Ciudadanas. Disponible en: www.mineducacion.gov.co/cvn/1665/articles-75768_ archivo_pdf

- Nussbaum, M. (agosto 25 de 2016). Una carta para el pueblo colombiano. Medellín: U.de A. Noticias. Disponible en: http://www.udea.edu.co/wps/ portal/udea/web/inicio/udea-noticias/udea-noticia/!ut/p/z0/fYwxD4IwFIT_ igsjeRWx6kgcTIyDgzHQxTzaRp-WPpBi_PkWHYyLy-Xu8t2BghKUxwedMRB 7dDFXSp6Wq3U2LXKxEzKXopD7fL7INrPDUcAW1H8gPtC161QBSrMP9hm gbPke0A3GYiKw_00XbuzHjzrxHEgT9ol4rz0ZHqlv3bMma9BE3mOqMX617W Brx61mx01N6Bnam6peSw3Q3A!!/

- Ospina, H., Alvarado, S., Botero, P., Patiño, J. y Cardona, M. (2011). Experiencias Alternativas de acción política con participación de jóvenes en Colombia. Obtenido de: http://www.cinde.org.co/PDF/EXPERIENCIAS\%20ALTERNATIVAS.pdf. 
- Palacios, M. (1999). Agenda para la democracia y negoción con las guerrillas. En: Francisco Leal Buitrago. Los laberintos de la guerra. Utopías e incertidumbres sobre la paz. Bogotá: Tercer mundo.

- Registraduría Civil de la Nación. (2018). Censo electoral. Disponible en: https:// wsr.registraduria.gov.co/-Censo-Electoral,3661-.html.

- Salamanca, M., Rodríguez, M., Cruz, J., Ovalle, R., Pulido, M. y Molano, A. (2016). Guía para la implementación de la Cátedra de la Paz. Bogotá: Editorial Santillana.

- Toro, J. (2002). Comunicación y participación para la niñez y la familia. En: Camino hacia nuevas ciudadanías. Bogotá: Departamento Administrativo de Bienestar Social del Distrito. 